\title{
La Escala de Competencia y Resiliencia Parental para madres y padres en contextos de riesgo psicosocial
}

\author{
Juan C. Martín ${ }^{1 *}$, Eduardo Cabrera ${ }^{2}$, Jaime León $^{1}$ y María J. Rodrigo ${ }^{3}$ \\ 1 Universidad de Las Palmas de Gran Canaria \\ 2 Ayuntamiento de San Bartolomé de Tirajana (Gran Canaria) \\ 3 Universidad de La Laguna
}

\begin{abstract}
Resumen: Este artículo describe el proceso de construcción de una escala para evaluar la competencia y resiliencia parental en contextos de riesgo psicosocial. La investigación fue realizada con 63 profesionales de los servicios sociales municipales de Gran Canaria sobre 498 casos de familias en riesgo para analizar las propiedades psicométricas de la escala. La estructura factorial se obtuvo a través de la técnica de modelo de ecuaciones estructurales exploratorio (MESE) con rotación oblimin y el método de estimación de ponderación de mínimos cuadrados ajustado por la media y la varianza (WLSMW) para su confirmación. La escala para madres mostró una estructura de cinco factores con 44 ítems: Desarrollo Personal y Resiliencia, Organización Doméstica, Búsqueda de Apoyo, Competencias Educativas y Competencias Comunitarias. Estos factores muestran una buena consistencia interna con alfas ordinales entre .70 y $.97 . \mathrm{La}$ escala para padres mostró 4 factores con 32 ítems: Desarrollo Personal y Resiliencia, Competencias Educativas y Organización Doméstica, Competencias Comunitarias y Búsqueda de Apoyo. Estos factores muestran una buena consistencia interna con alfas ordinales entre .68 y .97. Los resultados sugieren que el cuestionario, en sus versiones para madres y padres, captura adecuadamente la especificidad de cada rol parental en contextos de riesgo y muestra unas propiedades psicométricas adecuadas que lo hacen recomendable para su uso en el ámbito de los servicios sociales.

Palabras claves: Evaluación de la competencia y resiliencia parental; roles maternos y paternos; familias en riesgo; análisis factorial exploratorio y
\end{abstract} confirmatorio.

\begin{abstract}
Title: The Parental Competence and Resilience Scale for mother and fathers in at-risk psychosocial contexts.

Abstract: This paper describes the development of a scale to assess parental competence and resilience in psychosocial risk contexts. The research was conducted with 63 professionals from the Gran Canaria social services, (Canary Islands, Spain) over 498 cases of at-risk families. The factor structure was obtained by Exploratory Structural Equation Modeling (ESEM) with oblimin rotation, and the estimation method using the Weighted Least Squares with moving measurement window (WLSMW) for confirmatory purposes. The mothers scale showed a structure of five factors with 44 items: Personal Development and Resilience, Household Organization, Seek of Support, Educational Competence, and Community Competence. These factors showed good internal consistency (ordinal alpha ranging from .70 to .97). The fathers scale consisted of 4 factors with 32 items: Personal Development and Resilience, Educational and Household Organization Competence, Community Competence, Seek of support. These factors showed good internal consistency with ordinal alpha ranging from .68 to .97 . The results suggested that the questionnaire, in its double version for mothers and fathers, captures the specificities of each parental role and showed sound psychometric properties that make it suitable for the social services field.

Key words: Assessment of parental competence and resilience; mother and father roles; at-risk families; exploratory and confirmatory factor analyses.
\end{abstract}

\section{Introducción}

El uso sistemático de los programas de educación parental en los servicios de atención a las familias es un fenómeno actualmente en auge dentro de las políticas europeas de apoyo a la parentalidad positiva (Daly, 2007). Según la Recomendación (2006) 19 del Consejo de Europa sobre políticas de apoyo al ejercicio positivo de la parentalidad, el término de parentalidad positiva se refiere «al comportamiento de los padres fundamentado en el interés superior del menor, que cuida, desarrolla sus capacidades, no es violento y ofrece reconocimiento y orientación que incluyen el establecimiento de límites que permitan el pleno desarrollo del menor». La Recomendación se propone conseguir que los Estados miembros sean conscientes de la necesidad de proporcionar a los padres y las madres los apoyos psico-educativos suficientes para cumplir sus importantes responsabilidades en la crianza y educación de sus hijos/as, especialmente en aquelos que viven en circunstancias de riesgo psicosocial.

En línea con esta tendencia preventiva de apoyo a las familias vulnerables, se vienen desarrollando en España programas de educación parental dirigidos a las familias usuarias

* Dirección para correspondencia [Correspondence address]: Juan Carlos Martín. Departamento de Educación. Universidad de Las Palmas de Gran Canaria. C/. Santa Juana de Arco, 1. 35004. Las Palmas (España). E-mail: imartin@dedu.ulpgc.es de los servicios sociales especializados, que pretenden conseguir cambios objetivos y subjetivos de mejora en el ejercicio de las funciones parentales (Rodrigo, Máiquez, Martín y Byrne, 2008; Rodrigo, Máiquez y Martín, 2010a, 2010b). La identificación y evaluación de las competencias parentales es una tarea central para poder analizar la eficacia de dichos programas a la hora de promover la mejora de dichas competencias. Asimismo, la evaluación de competencias parentales es imprescindible, desde los servicios de apoyo a las familias, para poder evaluar sus fortalezas y no solo sus debilidades y problemas cuando se están planificando los objetivos a conseguir en la intervención de caso y se proponen los apoyos psicoeducativos que éstas precisan para reforzar el rol parental.

Para realizar esta evaluación es importante clarificar qué se entiende por competencia parental y cuáles son aquellas competencias básicas para lograr una educación positiva de los menores. Basándonos en los modelos de competencia general de Waters y Sroufe (1983) y Masten y Curtis (2000), las definimos como aquel conjunto de capacidades que permiten a los padres y a las madres afrontar de modo flexible y adaptativo la tarea vital de ser padres, de acuerdo con las necesidades evolutivas y educativas de los hijos e hijas y con los estándares considerados como aceptables por la sociedad, y aprovechando todas las oportunidades y apoyos que les brindan los sistemas de influencia de la familia para desplegar 
dichas capacidades (Rodrigo et al. 2008; Rodrigo, Martín, Cabrera y Máiquez, 2009). Según estos autores las competencias parentales son el resultado de un ajuste entre las condiciones psicosociales en las que vive la familia, el escenario educativo que los padres o cuidadores han construido para realizar su tarea vital y las características del menor.

Son escasos los trabajos en los que se hace referencia a las áreas de competencia parental más importantes para el ejercicio de la parentalidad positiva. Siguiendo los trabajos pioneros de Azar (1998), Azar y Cote (2002), Reder, Duncan y Lucey (2003) recopilados por White (2005) y la propuesta de síntesis de Rodrigo et al. (2009) se proponen las siguientes áreas competenciales: Área Educativa (vg., mostrar calidez y afecto en las relaciones con los hijos); Agencia Parental ( $v g$., sentirse eficaz y capaz como progenitor); Autonomía Personal y Búsqueda de Apoyo (vg., búsqueda de ayuda de personas significativas cuando tiene problemas con los hijos); y Área de desarrollo personal ( $v g$., responder a múltiples tareas y retos). Tratándose de familias en situación de riesgo psicosocial, el modelo se enriquece con algunos rasgos característicos de la capacidad resiliente tales como flexibilidad, cohesión, reflexión u optimismo vital ante la adversidad (Luthar, 2003; Rodrigo et al., 2010b; Walsh, 2004). A partir de este modelo teórico nos hemos propuesto elaborar una escala para evaluar las competencias parentales que pueda ser utilizada por los profesionales encargados de evaluar y apoyar a los padres y a las madres en su tarea parental.

En los últimos años se han ido publicando modelos de evaluación familiar en los sistemas de protección de menores de diversos países que incluyen las competencias parentales como un componente a tener en cuenta a la hora de tomar decisiones en la vida de los menores y proponer los objetivos de la intervención (White, 2005). Citemos entre los más relevantes para evaluar capacidades parentales el Framework for the Assessment of Children in Need and their Families (FACNF) desarrollado en Gran Bretaña (Department of Health et al. 2000) y que ha sido adoptado con éxito en numerosos países del ámbito anglosajón (Rose, 2010); el Comprehensive Family Assessment Guidelines for Child Welfare desarrollado por el $\mathrm{Na}$ tional Child Welfare Resource Center for Family-Centered Practice en Estados Unidos (Schene, 2005); el Standardized assessment of parenting desarrollado por el New Zealand Children, Young Persons, and Their Families Services (Barber y Delfabbro, 2000) y el reciente modelo basado en el buen trato y la resiliencia parental desarrollado por Barudy y colaboradores en el ámbito iberoamericano (Barudy y Danthagnan, 2010a). El FACNF propone tres dominios que son cruciales en la protección del menor: Las necesidades evolutivas del menor, las capacidades de los padres o cuidadores y los factores ambientales y familiares. Con respecto a las competencias, que es el objeto de nuestro estudio, el FACNF señala la capacidad de los padres para ofrecer cuidados básicos y seguridad, para ofrecer calidez emocional, estimular el desarrolo del menor, orientar y establecer límites y proporcionar un ambiente estable. El Comprehensive Family Assessment Guidelines for Child Welfare señala, entre otras, la capacidad de los pro- genitores para aceptar la responsabilidad sobre sus conductas y su motivación de cambio, la capacidad de conseguir que el niño vaya al colegio, la capacidad para controlar la expresión de ira, la capacidad para formar y mantener relaciones saludables y las habilidades de solución de problemas. El Standardized Assessment of Parenting evalúa la capacidad parental para proporcionar cuidados básicos que satisfagan las necesidades infantiles, evalúa el funcionamiento del sistema familiar, las prácticas positivas y negativas de los padres y las técnicas de resolución de conflictos con los hijos e hijas. Por último, el modelo desarrollado por Barudy y Danthagnan (2010a) diferencia entre las capacidades parentales fundamentales (apego y empatía) y las habilidades parentales, que son los modelos de crianza y la habilidad para participar en redes sociales y la utilización de recursos comunitarios. Aunque recomienda una serie de instrumentos aún no dispone de datos sobre sus propiedades psicométricas (Barudy y Dantagnan, 2010b)

Además de estos sistemas de evaluación familiar más generales, también existen instrumentos con propiedades psicométricas adecuadas que evalúan algunas de las áreas enumeradas más arriba. Algunos instrumentos con versión en castellano son: el instrumento Child Well-being Scale (Magura y Moses, 1986) traducida y validada por De Pául y Arruabarrena (1999) evalúa diversos aspectos como el cuidado parental para la satisfacción de necesidades infantiles, la disposición parental y el trato que recibe el niño que garantizan el bienestar infantil en el hogar. Johnson y colaboradores (2006), a partir de una revisión de 85 instrumentos para evaluar el funcionamiento familiar, señalaron el North Carolina Family Assessement Scale (NCFAS) (Reed-Ashcraft, Kirk y Fraser, 2001) como uno de los instrumentos con mejores cualidades para ser usado en los Servicios Sociales que, además, cuenta con una traducción castellana (Gómez, 2010). Esta escala evalúa, por ejemplo, la supervisión del niño y las prácticas disciplinarias. Otros instrumentos evalúan el área de agencia parental: el cuestionario de Sentido de Competencia Parental (Johnston y Mash, 1989) mide la Eficacia y la Satisfacción parental (versión castellana de Menéndez, Jiménez e Hidalgo (en prensa); la escala de Maternal Sense of Competence del cuestionario de Parenting Stress Index (Abidin, 1983) (versión castellana de Díaz-Herrero, Brito, López, Pérez-López y Martínez-Fuentes, 2010) evalúa también la percepción de la competencia parental; la Escala de competencia parental percibida (Bayot y Hernández, 2008) mide la implicación personal, la dedicación personal, el ocio compartido, el asesoramiento/orientación y la asunción del rol parental.

Los instrumentos que acabamos de citar sólo evalúan algunas áreas de las competencias parentales y están basados en muchos casos en el punto de vista de los padres y de las madres. Esto último supone una fuente importante de sesgos por tres razones: 1) La autopercepción de la competencia parental difiere mucho en función del estatus de riesgo de la familia ya que las familias en riesgo psicosocial tienden a sobrevalorar sus competencias y las familias normalizadas tienden a infravalorarlas (Rodrigo, Máiquez, Correa, Martín y 
Rodríguez, 2006); 2) la evaluación de las competencias parentales debe tener en cuenta el punto de vista del profesional que va a tomar decisiones importantes sobre la vida de los menores. También es importante su punto de vista para identificar las necesidades formativas o de intervención que pudiera necesitar la unidad familiar a fin de mejorar las tareas parentales; y 3) es importante conocer las competencias del padre y de la madre lo cual es difícil cuando los padres son los informantes ya que la figura paterna en las familias en riesgo, de existir suele ser poco accesible, más inestable y estar muy poco implicada en la educación de los hijos e hijas (Lerman, 2010; Lewis y Lamb, 2003; Minuchin, Colapinto y Minuchin, 2000).

Por todo ello, en esta investigación nos proponemos elaborar una escala para que los profesionales evalúen el cuadro completo de áreas de competencia y resiliencia parental tanto en los padres como en las madres en contextos de riesgo psicosocial y analizar sus propiedades psicométricas.

\section{Método}

\section{Participantes}

La muestra estuvo constituida por un total de 498 unidades familiares que estaban siendo atendidas en los Servicios de atención a la infancia y la familia de los 21 municipios de la isla de Gran Canaria en el año 2010-2011. De las 498 unidades familiares el $48.9 \%$ eran monoparentales y el $51.1 \%$ biparentales. Por ello contábamos con un $95.9 \%$ de madres y un $56.8 \%$ con padres. Respecto a la cronicidad de la problemática presentada (más de dos años siendo atendidas en los servicios sociales) el 59.9\% eran crónicas y el $40.1 \%$ eran no crónicas. De las familias evaluadas, el $46.5 \%$ presentaban problemática transgeneracional (al menos dos generaciones han sido atendidas en los Servicios Sociales) y el 53.5\% no tenían esta problemática. El 30.3\% vivían en zonas rurales y el $69.7 \%$ vivían en zonas urbanas. En cuanto a la edad de la madre el $23.9 \%$ tenía entre $18-30$ años, el $21.4 \%$ entre $31-36$ años, el $29.2 \%$ entre $37-42$ años y el $25.5 \%$ más de 43 años. En cuanto a la edad del padre, el 23.3\% tenía entre 20-35 años, el $23.9 \%$ tenía entre 36-41 años, el 31.5\% tenía entre
42-47 años y el 21.2\% 48 años o más. La edad media de las madres fue de 36.9 años $(D T=8.21)$ y la edad media de los padres fue de 41.7 años $(D T=8.87)$. El $63.4 \%$ de los padres no tenían estudios finalizados y el $36.6 \%$ de los padres habían cursado ESO o Bachillerato. En cambio, el 58.6\% de las madres no tenían estudios y el $41.3 \%$ habían terminado ESO o Bachillerato. El $68.3 \%$ de las familias recibían ayudas económicas. Por último, la media del número de hijos era de 1.62 y la desviación típica de 1.29.

\section{Desarrollo de la Escala de Competencia y Resilien- cia Parental}

Se partió de las cinco áreas competenciales descritas por Rodrigo et al. (2008) y Rodrigo, et al. (2009) y reseñadas más arriba. Se llevaron a cabo dos grupos de discusión con los profesionales que trabajan en varios servicios sociales de Gran Canaria para analizar dichas competencias, como resultado de los cuales el modelo se amplió a diez áreas competenciales y 62 habilidades o capacidades englobadas en las mismas tras quitar el ítem AP17 que se confundía con el ítem AP5 (ver Tabla 1). Los ítems de la escala fueron elegidos en función de la relevancia y representatividad encontrada no sólo en los textos académicos sino también según la experiencia práctica en la evaluación de dichas competencias por parte de los profesionales de los servicios de atención a menores y familias en el ámbito de los Servicios Sociales (Elousa, 2003). Así se formó la primera versión de la escala con 62 ítems en los cuales el profesional debía valorar con una escala tipo Likert de 5 puntos (nada a mucho) en qué medida el padre o la madre poseía dichas competencias. Se eligió un formato de respuesta de cinco alternativas, dada las evidencias empíricas que señalan que el número óptimo de alternativas que están asociadas a una mejora de la fiabilidad y la validez de las pruebas se sitúan entre 4 y 7 (Lozano, García-Cueto y Muñiz, 2008) Para facilitar la comprensión de los ítems se elaboró un manual en el que cada competencia iba acompañada de una breve referencia conceptual y una descripción orientativa sobre qué significa una puntuación baja o una puntuación alta. La versión inicial era igual para padres y madres.

Tabla 1. Conjunto inicial de áreas competenciales requeridas para la tarea de ser padres y madres en contextos de riesgo psicosocial. Áreas competenciales Agencia parental Se siente eficaz y capaz como progenitor Siente que posee control sobre los acontecimientos de la vida Se siente capaz para promover y llegar a acuerdos con la pareja Percibe su rol parental de manera ajustada

Reconoce la importancia de los progenitores en el bienestar del menor

Promoción de la salud Se preocupa por la higiene de los menores Se preocupa de la propia higiene

Hace ejercicio físico / deporte

Promueve el ejercicio físico en los menores

Hace un uso adecuado de la medicación (si lo necesitara)

Muestras estrategias de afrontamiento ante situaciones de estrés Prepara comidas saludables 


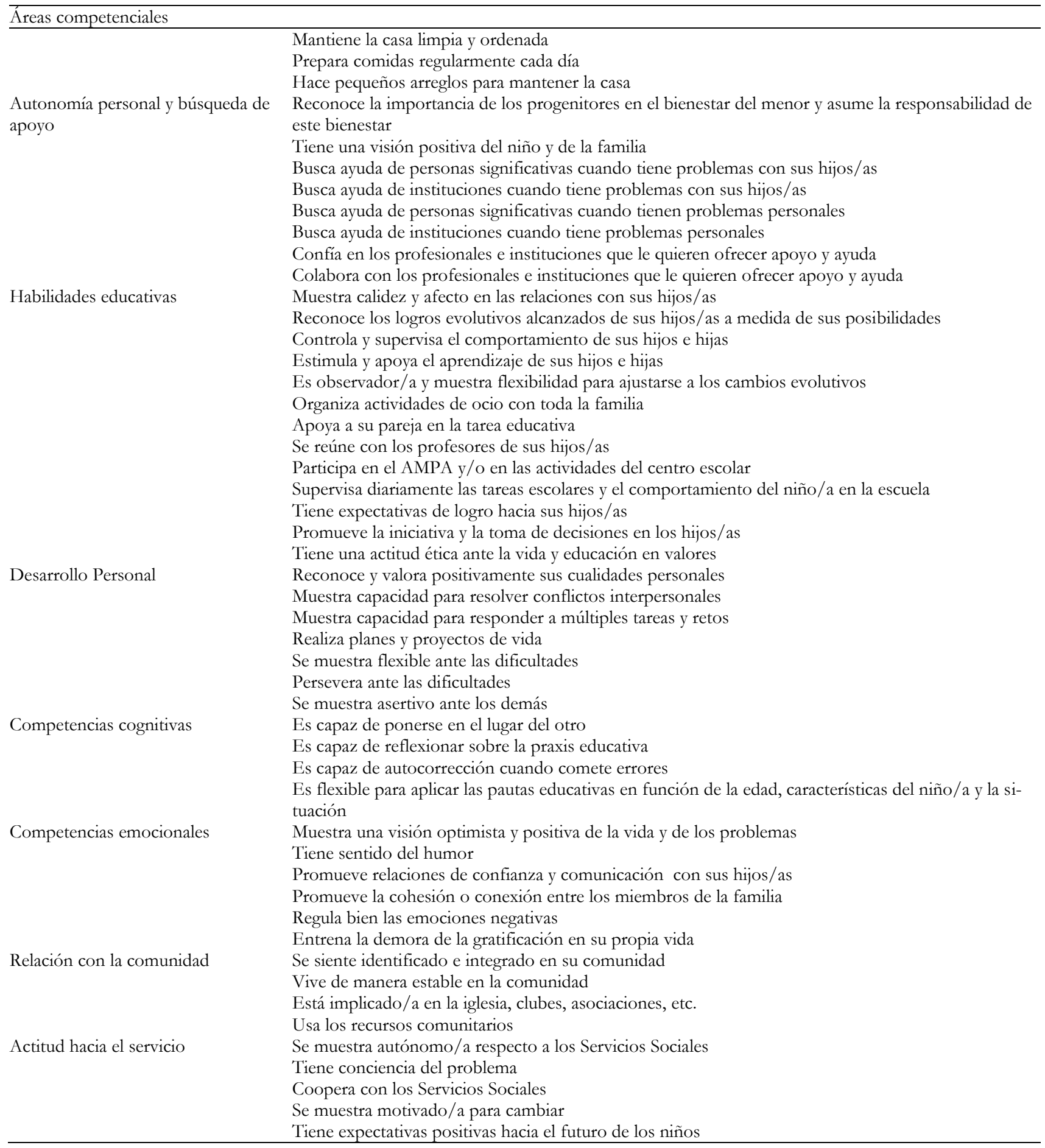

\section{Procedimiento}

Tras lograr el apoyo institucional de la Dirección General de Protección al Menor y la Familia del Gobierno Autónomo de Canarias y de la Consejería de Política Social y Sociosanitaria del Cabildo de Gran Canaria, se invitó a todos los municipios de la isla de Gran Canaria a participar en una reunión con el objeto de presentar la investigación e invitar a los distintos equipos técnicos de atención a la infancia y la familia a participar en la misma. En dicha reunión con los técnicos municipales se les entregó una carta de adhesión a la investigación (que debía firmar el político responsable de servicios sociales), un resumen de la investigación y el cronograma del proceso de investigación. El 100\% de los muni- 
cipios de la isla se adhirieron a la investigación y autorizaron a participar a 63 técnicos. De estos, el $88.9 \%$ eran mujeres y el $11.1 \%$ hombres. Respecto a la formación académica, el $49 \%$ eran trabajadores sociales, el $27.5 \%$ eran educadores sociales, el $11.8 \%$ eran psicólogas, el $8 \%$ eran maestras y el $4 \%$ eran pedagogas. La edad media de los técnicos fue de 34.8 años $(D T=6.5)$ y por término medio tenían 6 años de experiencia en el trabajo con familias en riesgo $(D T=4.2)$. El profesional que participó en la investigación y que tenía que cumplimentar los datos de las familias debía cumplir dos condiciones: que estuviera entrenado en el uso del cuestionario asistiendo al curso de formación impartido por los investigadores (formación sobre el uso del cuestionario y el modelo teórico que sustentaba la investigación) y que hubiera tenido al menos tres encuentros con la familia que iba a evaluar.

Ya en el curso de formación se les presentó a los profesionales el cuestionario y el manual de uso. Durante dos días se formaron teóricamente y se hicieron prácticas con casos reales para aprender a utilizar la escala. A partir de la formación se entregó a cada equipo técnico el manual y la escala y se les informó sobre el procedimiento para hacer el seguimiento de la implementación de los cuestionarios. Se utilizaron cuatro sistemas de supervisión del proceso de recogida de datos: creación de un foro en internet, correo electrónico, llamadas telefónicas y, para llevar un seguimiento de la investigación, visitas del equipo investigador a los municipios.

Una vez recogidos los cuestionarios y resueltas las incidencias se entregó a cada uno de los participantes un diploma acreditativo de la formación recibida y asimismo se les entregó un certificado de participación en la investigación y se les invitó a una futura presentación de los resultados.

\section{Análisis de datos}

Para determinar la estructura factorial de la escala utilizamos la técnica multivariada, modelo de ecuaciones estructurales exploratorio (MESE), desarrollado por Asparouhov y Muthen (2009). La ventaja principal de esta técnica es combinar el análisis factorial exploratorio (AFE) con el análisis factorial confirmatorio (AFC), además no requiere que el peso factorial de los ítems en los demás factores sea cero, por lo que el cálculo de los índices de ajuste y de las correlaciones entre variables latentes son más precisos (Asparouhov y Muthen, 2009; Marsh H.W., Muthén B., Asparouhov T., Lüdtke, O. Robitzsch A. Morin A. y Trautwein U., 2009).

Respecto al método de rotación utilizado, aunque Marsh et al. (2009) señalan que es una cuestión todavía abierta, decidimos utilizar una rotación geomin ya que Asparouhov y Muthen (2009) observaron que ésta se comporta adecuadamente cuando se conoce poco de la estructura factorial a estudiar. Más especificamente, utilizamos el geomin oblícuo, ya que los métodos que implican rotaciones oblicuas, en las ciencias sociales, muestran relaciones entre factores más cercanas a la realidad (Brown, 2006; Schmitt, 2011).
En cuanto al método de estimación utilizado, al ser la escala tipo Likert, las variables observables son categóricas ordinales (Flora y Curran, 2004) y, por lo tanto, para estimar el valor de los parámetros y los índices de ajuste es más preciso utilizar un método de estimación que no requiere normalidad multivariada (Schmitt, 2011). Por ello, utilizamos el método de mínimos cuadrados ponderados ajustado por la media y la varianza (WLSMW, por sus siglas en inglés Weighted Least Squares Mean and Variance Adjusted). Si bien es cierto que este método no requiere de normalidad multivariada, algunos autores han señalado que valores extremos de asimetría puede afectar a la estimación de los parámetros (Kline, 2011; Muthén, du Toit y Spisic, 1997). El rango de asimetría de los ítems fue, en valores absolutos, entre .001 y 3.257.

Además el hecho de que un técnico evaluó a varios sujetos viola el supuesto de independencia, esto puede inflar el valor de $\chi^{2}$ e infraestimar los errores típicos (Stapleton, 2006). Para corregir esto, los parámetros fueron estimados maximizando una función logarítmica ponderada y los errores típicos utilizando un estimador tipo sandwich (Muthén y Muthén, 1998-2010). Para analizar el ajuste del modelo al patrón de los datos utilizamos la prueba de $\chi^{2}$, la ratio $\chi^{2} / g l$, el índice RMSE $A$, el índice de Tucker-Lewis (TLI), el índice de ajuste comparativo (CFI) y el residuo ponderado cuadrático medio ( $W R M R)$.

Para decidir el número de factores seguimos varios criterios, en primer lugar el sentido teórico de los mismos, en segundo lugar, como indicador de discriminación de los factores se prestó atención al número de factores con al menos tres ítems significativos en el factor esperado y que fueran significativos $(\mathrm{NC}=95 \%)$ únicamente en uno o dos factores y, por último, se atendió a los índices de ajuste, teniendo en cuenta que seguir este criterio puede llevar a aceptar más factores de los necesarios (Hayashi, Bentler y Yuan, 2007). Una vez determinado el número de factores, se descartaron los ítems con pesos inferiores a .30 y aquellos cuya diferencia de pesos entre dos factores era menor a 15 .

A continuación se analizaron las evidencias de fiabilidad, para ello utilizamos el alfa ordinal en lugar del habitual alfa de Cronbach, ya que este último requiere que el peso factorial sea igual para todos los ítems (Yang y Green, 2010) y que los datos sean continuos (Elosua y Zumbo, 2008). Los valores perdidos se estimaron utilizando el método WLSMV (Asparouhov y Muthén, 2010). Para realizar los estadísticos descriptivos se utilizó el software $\mathrm{R}$ ( $\mathrm{R}$ Core Development Team, 2011); para el MESE, el Mplus 6.11 (Muthén y Muthén, 1998-2011) y, por último, para estimar el alfa ordinal se utilizó el Microsoft Excel.

Normalmente se considera que CMIN/DF con valores inferiores a 2 indican un buen ajuste (Tabachnick y Fidell, 2007). El índice CFI, TLI varía entre 0 y 1 , con 0 indicando ausencia de ajuste y 1 ajuste óptimo. Valores de .95 o superiores son considerados excelentes, y valores superiores a .90 sugieren un ajuste aceptable del modelo a los datos. El índice RMSEA es considerado óptimo cuando sus valores son de .05 o inferiores y aceptables en el rango .08-.05 ( Hu y Ben- 
tler, 1995; Byrne, 2010). En este estudio se consideró que el modelo tendría un buen ajuste si $\mathrm{RMSE} A \leq .05$; CFI $y$ TLI $\geq .95$ y $C M I N / D F \leq .2$. $y$ WRMR $\leq 1$

\section{Resultados}

\section{Estructura factorial de la escala materna}

Para decidir la estructura factorial se siguieron los criterios descritos anteriormente. En la Tabla 2 se puede observar que la estructura factorial en la que coincide el número de factores con al menos tres ítems significativos en un factor $y$ que solo son significativos en uno o dos factores es con la solución de cinco factores, es decir a más factores menor discriminación, ya que los ítems son significativos en tres o más factores. Respecto a los índices de ajuste, se puede observar que a mayor número de factores, mejor ajuste, datos que están en consonancia con los resultados de Hayashi et al. (2007). Asparouhov y Muthen (2009) han observado con puntuaciones continuas que si añadir un factor disminuye el SRMR en .001 o menos, no merece la pena mantener la estructura con más factores. En este estudio, al ser con variables categóricas se atendió al valor del RMSE $A$ y así, a raíz de los datos, podríamos descartar la opción de más de cinco factores. Finalmente, atendiendo a la discriminación de los factores, al valor de RMSEA y al sentido teórico, se optó por la estructura de 5 factores. Seguidamente se eliminaron los ítems con pesos menores de .30 y los que tenían una diferencia menor de .15 en dos factores.

Tabla 2. Escala materna. Número de factores con tres ítems significativos (95\%) en un factor y que no son significativos en más de uno o dos factores e índices de ajuste en función del número de factores en cada extracción.

\begin{tabular}{|c|c|c|c|c|c|c|c|c|}
\hline Factores & Fact/Ítems & $\chi^{2}$ & $g l$ & RMSEA & $90 \%$ & $C F I$ & $T L I$ & $W \mathrm{R} M \mathrm{R}$ \\
\hline 1 & 1 & 3219.845 & 1890 & .038 & $.036-.040$ & .92 & .917 & 2.307 \\
\hline 2 & 2 & 2744.639 & 1828 & .032 & $.030-.034$ & .945 & .941 & 1.781 \\
\hline 3 & 3 & 2420.169 & 1767 & .027 & $.025-.030$ & .961 & .956 & 1.394 \\
\hline 4 & 4 & 2189.752 & 1707 & .024 & $.021-.027$ & .971 & .967 & 1.159 \\
\hline 5 & 5 & 2044.166 & 1648 & .022 & $.019-.025$ & .976 & .972 & 1.009 \\
\hline 6 & 3 & 1935.564 & 1590 & .021 & $.018-.024$ & .979 & .974 & .894 \\
\hline 7 & 0 & 1834.878 & 1533 & .020 & $.016-.023$ & .982 & .977 & .807 \\
\hline 8 & 4 & 1730.714 & 1477 & .019 & $.015-.022$ & .985 & .980 & .725 \\
\hline 9 & 0 & 1640.509 & 1422 & .018 & $.013-.022$ & .987 & .982 & .648 \\
\hline 10 & 0 & 1548.837 & 1368 & .016 & $.011-.021$ & .989 & .984 & .586 \\
\hline
\end{tabular}

Nota. Fact/Ítems = Número de factores con tres o más ítems significativos (95\%) en un factor y que no son significativos en más de uno o dos factores.

La solución factorial obtenida estuvo formada por 5 factores, donde los pesos factoriales en sus respectivos factores oscilaron entre .361 y .898 (Ver Tabla 3). El primer factor, organización doméstica, determinado por 6 ítems; el segundo factor, competencias educativas, formado por 13 ítems; el tercer factor, desarrollo personal y resiliencia, estaba formado por 14 ítems. El cuarto factor, búsqueda de apoyo, formado por 5 ítems. Finalmente, el quinto factor denominado integración comunitaria estaba formado por 6 ítems.

Tabla 3. Pesos factoriales para la versión definitiva de la escala materna.

\begin{tabular}{|c|c|c|c|c|c|}
\hline Items & F1 & F2 & F3 & F4 & F5 \\
\hline Se ocupa de la propia higiene & .898 & .043 & -.009 & -.088 & .000 \\
\hline Mantiene la casa limpia y ordenada & .839 & -.002 & .032 & .031 & -.021 \\
\hline Prepara comidas regularmente cada día & .809 & -.079 & .080 & .055 & .086 \\
\hline Se ocupa de la higiene de los menores & .807 & .240 & -.046 & .000 & -.050 \\
\hline Prepara comidas saludables & .759 & .041 & .129 & .037 & -.061 \\
\hline Hace pequeños arreglos para mantener la casa & .527 & .039 & .201 & .020 & .032 \\
\hline Muestra calidez y afecto en las relaciones con sus hijos/as & .006 & .905 & -.014 & .046 & -.249 \\
\hline Tiene expectativas de logro hacia sus hijos/as & .005 & .856 & .041 & -.264 & .137 \\
\hline Reconoce los logros evolutivos alcanzados de sus hijos/as a medida de sus posibilidades & -.009 & .852 & .077 & .061 & -.147 \\
\hline Promueve relaciones de confianza y comunicación con sus hijos/as & -.030 & .809 & .079 & .056 & -.077 \\
\hline Promueve la iniciativa y la toma de decisiones en los hijos/as & .004 & .804 & .097 & -.236 & .096 \\
\hline Tiene una visión positiva del niño y de la familia & -.059 & .791 & .012 & .055 & -.123 \\
\hline Supervisa diariamente las tareas escolares y el comportamiento del niño/a en la escuela & .099 & .737 & -.037 & -.115 & .194 \\
\hline Estimula y apoya el aprendizaje de sus hijos e hijas & .113 & .705 & .079 & -.037 & .163 \\
\hline Controla y supervisa el comportamiento de sus hijos e hijas & .159 & .673 & .001 & .032 & .052 \\
\hline Promueve la cohesión o conexión entre los miembros de la familia & -.024 & .606 & .148 & .172 & .006 \\
\hline Se reúne con los profesores de sus hijos/as & .155 & .519 & -.051 & .082 & .196 \\
\hline Organiza actividades de ocio con toda la familia & .160 & .497 & .009 & .058 & .130 \\
\hline Participa en el AMPA y/o en las actividades del centro escolar & .059 & .398 & -.126 & .054 & .380 \\
\hline Muestra capacidad para responder a múltiples tareas y retos & .115 & -.077 & .868 & .018 & .043 \\
\hline Se muestra flexible ante las dificultades & .001 & -.016 & .862 & .081 & -.063 \\
\hline
\end{tabular}




\begin{tabular}{|c|c|c|c|c|c|}
\hline Ítems & F1 & F2 & F3 & $\mathrm{F} 4$ & F5 \\
\hline Muestra capacidad para resolver conflictos interpersonales & .001 & .105 & .802 & -.027 & -.015 \\
\hline Muestras estrategias de afrontamiento ante situaciones de estrés & .058 & .132 & .754 & -.024 & -.129 \\
\hline Persevera ante las dificultades & .067 & -.009 & .740 & .102 & .048 \\
\hline Se muestra asertivo ante los demás & -.058 & .155 & .696 & .035 & -.021 \\
\hline Siente que posee control sobre los acontecimientos de la vida & .004 & .152 & .665 & -.020 & -.002 \\
\hline Muestra una visión optimista y positiva de la vida y de los problemas & -.072 & .155 & .664 & -.034 & .030 \\
\hline Realiza planes y proyectos de vida & .142 & .082 & .656 & -.029 & .113 \\
\hline Reconoce y valora positivamente sus cualidades personales & .065 & -.079 & .640 & -.229 & .115 \\
\hline Regula bien las emociones negativas & -.040 & .348 & .622 & .001 & -.083 \\
\hline Es capaz de autocorrección cuando comete errores & -.002 & .249 & .550 & .207 & .024 \\
\hline Hace un uso adecuado de la medicación (si lo necesitara) & .191 & -.034 & .477 & .017 & .037 \\
\hline Entrena la demora de la gratificación en su propia vida & -.028 & .289 & .476 & .021 & .049 \\
\hline Busca ayuda de personas significativas cuando tienen problemas personales & .033 & .117 & -.106 & .842 & .005 \\
\hline Busca ayuda de instituciones cuando tiene problemas personales & -.006 & -.023 & .052 & .831 & -.037 \\
\hline Busca ayuda de instituciones cuando tiene problemas con sus hijos/as & .034 & -.062 & .082 & .794 & .181 \\
\hline Busca ayuda de personas significativas cuando tiene problemas con sus hijos/as & .046 & .128 & -.027 & .750 & .098 \\
\hline Confía en los profesionales e instituciones que le quieren ofrecer apoyo y ayuda & .014 & .091 & .230 & .573 & .029 \\
\hline Está implicado/a en la iglesia, clubes, asociaciones, etc. & -.020 & .019 & .038 & .161 & .628 \\
\hline Usa los recursos comunitarios & -.133 & .053 & .142 & .241 & .604 \\
\hline Se siente identificado e integrado en su comunidad & -.039 & -.001 & .035 & .057 & .569 \\
\hline Hace ejercicio físico / deporte & .258 & .041 & .007 & -.036 & .500 \\
\hline Vive de manera estable en la comunidad & -.036 & .021 & -.038 & -.062 & .491 \\
\hline Promueve el ejercicio físico en los menores & .114 & .210 & .070 & .041 & .361 \\
\hline
\end{tabular}
Nota. F1= Organización Doméstica; F2= Competencias educativas; F3= Desarrollo Personal y Resiliencia; F4= Búsqueda de Apoyo; F5= Integración comunitaria.

\section{Fiabilidad}

La consistencia interna de la versión final de la escala materna fue estimada mediante el coeficiente alfa ordinal. Los resultados mostraron una fiabilidad adecuada tanto de la escala global de 44 ítems (.97) como de las 5 dimensiones: organización doméstica, (.89); competencias educativas, (.93); desarrollo personal y resiliencia, (.92); búsqueda de apoyo, (.87); y competencia comunitaria, (.70).

\section{Estructura factorial de la escala paterna}

En la Tabla 4 se puede observar que la estructura factorial en la que coincide el número de factores con tres o más ítems significativos en un factor y que no son significativos en más de uno o dos factores, es con la propuesta de 4 factores. Respecto a los índices de ajuste, igual que con la escala materna, a mayor número de factores, mejores índices de ajuste. Respecto al RMSEA, descartamos la opción de más de cinco factores. Finalmente, atendiendo a la discriminación de los factores y al sentido teórico de los ítems, se optó por la estructura de 4 factores. Seguidamente se eliminaron los ítems con pesos menores de .30 y los que tenían una diferencia menor de .15 en dos factores.

Tabla 4. Escala paterna. Número de factores con tres o más ítems significativos (95\%) en un factor y que no son significativos en más de uno o dos factores e índices de ajuste en función del número de factores en cada extracción.

\begin{tabular}{|c|c|c|c|c|c|c|c|c|}
\hline Factores & Fact/Ítems & $\chi^{2}$ & $g l$ & RMSEA & $90 \%$ & $C F I$ & $T L I$ & $W \mathrm{R} M \mathrm{R}$ \\
\hline 1 & 1 & 2650.611 & 1890 & .034 & $.031-.037$ & .958 & .956 & 1.730 \\
\hline 2 & 2 & 2397.295 & 1828 & .030 & $.270-.033$ & .968 & .966 & 1.419 \\
\hline 3 & 3 & 2248.068 & 1767 & .028 & $.024-.032$ & .973 & .971 & 1.235 \\
\hline 4 & 4 & 2102.159 & 1707 & .026 & $.022-.030$ & .978 & .975 & 1.090 \\
\hline 5 & 3 & 1969.851 & 1648 & .024 & $.019-.028$ & .982 & .976 & .962 \\
\hline 6 & 3 & 1877.885 & 1590 & .023 & $.018-.027$ & .984 & .980 & .875 \\
\hline 7 & 3 & 1788.738 & 1533 & .022 & $.017-.026$ & .986 & .983 & .791 \\
\hline 8 & 0 & 1695.751 & 1477 & .021 & $.015-.025$ & .988 & .984 & .719 \\
\hline 9 & 2 & 1619.218 & 1422 & .020 & $.014-.025$ & .989 & .985 & .656 \\
\hline 10 & 1 & 1541.903 & 1368 & .019 & $.013-.024$ & .990 & .986 & .604 \\
\hline
\end{tabular}

Nota. Fact/Ítems = Número de factores con tres o más ítems significativos (95\%) en un factor y que no son significativos en más de uno o dos factores

El instrumento desarrollado quedó compuesto por 4 factores y 38 ítems, como se puede observar en la tabla 5, el pe- so factorial más bajo en su respectivo factor fue .400 y el más alto .876 . El primer factor denominado competencias educa- 
tivas y organización doméstica, formado por 7 ítems; el segundo factor desarrollo personal y resiliencia estaba formado por 14 ítems. El tercer factor, búsqueda de apoyo, estaba formado por
7 ítems. Finalmente, el cuarto factor denominado integración comunitaria estaba formado por 4 ítems.

Tabla 5. Pesos factoriales para la versión definitiva de la escala paterna.

\begin{tabular}{|c|c|c|c|c|}
\hline Ítems & F1 & F2 & F3 & F4 \\
\hline Prepara comidas regularmente cada día & .868 & .04 & .002 & -.364 \\
\hline Prepara comidas saludables & .777 & .200 & -.097 & -.306 \\
\hline Supervisa diariamente las tareas escolares y el comportamiento del niño/a en la escuela & .776 & -.029 & .142 & -.031 \\
\hline Se reúne con los profesores de sus hijos/as & .722 & -.135 & .288 & -.002 \\
\hline Controla y supervisa el comportamiento de sus hijos e hijas & .617 & .106 & .087 & .116 \\
\hline Se ocupa de la higiene de los menores & .483 & .216 & .084 & .111 \\
\hline Organiza actividades de ocio con toda la familia & .400 & .231 & .121 & .144 \\
\hline Se muestra flexible ante las dificultades & -.033 & .885 & .055 & .001 \\
\hline Muestra capacidad para resolver conflictos interpersonales & .055 & .870 & -.001 & -.066 \\
\hline Se muestra asertivo ante los demás & -.047 & .850 & .069 & -.145 \\
\hline Regula bien las emociones negativas & .025 & .840 & .032 & -.113 \\
\hline Tiene sentido del humor & -.064 & .835 & -.051 & .030 \\
\hline Muestra una visión optimista y positiva de la vida y de los problemas & .032 & .806 & -.087 & .015 \\
\hline Es capaz de autocorrección cuando comete errores & .053 & .769 & .189 & -.013 \\
\hline Tiene una visión positiva del niño y de la familia & .109 & .653 & .024 & .085 \\
\hline Se siente capaz para promover y llegar a acuerdos con la pareja & -.009 & .628 & .061 & -.103 \\
\hline Persevera ante las dificultades & .155 & .627 & .020 & .125 \\
\hline Tiene expectativas positivas hacia el futuro de los niños & .155 & .624 & .023 & .149 \\
\hline Se muestra motivado/a para cambiar & .118 & .590 & .368 & -.037 \\
\hline Realiza planes y proyectos de vida & .343 & .551 & -.129 & .130 \\
\hline Reconoce y valora positivamente sus cualidades personales & .191 & .511 & -.223 & .018 \\
\hline Busca ayuda de instituciones cuando tiene problemas personales & .063 & -.086 & .810 & -.241 \\
\hline Colabora con los profesionales e instituciones que le quieren ofrecer apoyo y ayuda & -.038 & .366 & .763 & .024 \\
\hline Busca ayuda de instituciones cuando tiene problemas con sus hijos/as & .129 & .035 & .752 & .035 \\
\hline Confía en los profesionales e instituciones que le quieren ofrecer apoyo y ayuda & -.160 & .380 & .744 & .023 \\
\hline Coopera con los Servicios Sociales & .005 & .403 & .691 & -.019 \\
\hline Busca ayuda de personas significativas cuando tienen problemas personales & .037 & .072 & .651 & .018 \\
\hline Busca ayuda de personas significativas cuando tiene problemas con sus hijos/as & .077 & .184 & .592 & .048 \\
\hline Se siente identificado e integrado en su comunidad & -.027 & .236 & -.048 & .876 \\
\hline Vive de manera estable en la comunidad & .085 & -.035 & -.013 & .612 \\
\hline Usa los recursos comunitarios & .377 & -.013 & .202 & .485 \\
\hline Está implicado/a en la iglesia, clubes, asociaciones, etc. & .220 & .087 & .045 & .407 \\
\hline
\end{tabular}

\section{Fiabilidad}

El análisis de la consistencia interna, a través del alfa ordinal, mostró una buena fiabilidad tanto de la escala global de 32 ítems (.97) como de las 4 dimensiones: competencias educativas y organización doméstica (.84); desarrollo personal y resiliencia, (.94); búsqueda de apoyo, (.88); e integración comunitaria, (.68).

\section{Discusión}

El objetivo de este estudio fue elaborar una escala de evaluación de competencia y resiliencia parental, reportada por profesionales de los servicios sociales y examinar sus propiedades psicométricas. Para ello se han llevado a cabo análisis con modelos de ecuaciones estructurales exploratorio y pruebas de consistencia interna. Los resultados obtenidos han aportado evidencia sobre la idoneidad de dicha escala en versión materna y paterna. En particular, los resultados muestran la idoneidad de una estructura de 5 y 4 factores pa- ra las versiones de la prueba (madre y padre), mostrando unos índices estadísticos de ajuste óptimos (Tabachnick y Fidell, 2007; Hu y Bentler, 1995; Byrne, 2010) y unos índices de consistencia interna adecuados tanto para las escalas globales como para cada uno de los factores.

La escala, en su versión para madres y padres, parece cubrir satisfactoriamente las competencias parentales que señalan los modelos británico (Department of Health et al., 2000), americano (Shene, 2005) e iberoamericano (Barudy y Danthagnan, 2010a). Así, por ejemplo, el Comprehensive Family Assessment Guidelines for Child Welfare de Estados Unidos incluye la supervisión escolar, la capacidad para controlar la expresión de ira, la capacidad para formar y mantener relaciones saludables, patrones positivos de solución de problemas, competencias todas ellas incluidas en nuestra escala. Asimismo, nuestra escala incluye las capacidades educativas ligadas a los modelos de crianza y las de integración comunitaria señaladas en el modelo de Barudy y colaboradores (Barudy y Danthagnan et al., 2010a) así como aquellas compe- 
tencias emotivas, cognitivas y conductuales que permiten construir el apego. También evalúa aquellas competencias que se consideran básicas desde el modelo británico (Department of Health et al., 2000) tales como la capacidad de asegurar las necesidades básicas del menor como la alimentación y la higiene, las competencias que permiten garantizar la seguridad y orientación del menor como el control y supervisión del comportamiento infantil y la organización de actividades de ocio con toda la familia. Como el modelo de Nueva Zelanda evalúa la capacidad parental para proporcionar cuidados básicos que satisfagan las necesidades infantiles y evalúa las competencias educativas (Barber y Delfabbro, 2000). Por último, compite ventajosamente con aquellos instrumentos que evalúan competencias únicamente centradas en la agencia parental como, por ejemplo, la escala de competencia parental percibida (Bayot y Hernández, 2008), entre otras.

De hecho, analizando el contenido de las escalas se observa que aparecen representadas todas las áreas competenciales del modelo inicial mucho más comprehensivo y amplio que los anteriores (Rodrigo et al., 2009), así como del modelo ampliado reflejado en el Cuadro 1 que constituyó la base para construir la versión inicial de la misma. Los resultados indican que, en general, padres y madres poseen competencias similares aunque en las madres se organizan en cinco factores y en cuatro factores en el caso de los padres. Así, en el caso de las madres aparece separada la organización doméstica y las competencias educativas, lo que da idea de la separación funcional del rol de organizadora del hogar y del educativo en las madres, mientras que en el caso del padre quedan ambas funciones fundidas en un solo factor. El resto de factores permanece bastante semejante: desarrollo personal y resiliencia, búsqueda de apoyo e integración comunitaria.

Sin embargo, aparecen algunas diferencias muy interesantes entre los progenitores. Además de permanecer separadas las competencias educativas y de organización doméstica en las madres, esta última área se enriquece con habilidades que sugieren el mayor peso de éstas en la estructuración de la vida cotidiana. Caracterizan a las madres su mayor participación en la limpieza y orden de la casa así como la higiene y los arreglos para mantener la casa. También su papel en la capacidad para "crear familia" asumiendo la promoción de la calidez, afecto y comunicación en las relaciones con los hijos, la participación en el desarrollo escolar y personal de estos así como la cohesión familiar. En los padres se ve el control por la higiene y la participación en la elaboración de comidas pero también se observan comportamientos de supervisión escolar y de regulación del comportamiento de los hijos e hijas. Todo ello refleja una realidad clave de estas familias que es el papel de timón organizador de la vida familiar que tienen las madres al ser éstas las que estructuran la vida cotidiana (Lerman, 2010; Minuchin et al. 2000; Rosenberg y Bradford, 2006; Yárnoz, 2006; Tamis-Lemonda y McFadden, 2010). Esta es una realidad que es bastante extensible a las familias normalizadas (Lewis y Lamb, 2003; Lamb, 2010). Pero la escala permite comprobar hasta qué punto se da la implicación del padre en la educación de los hijos algo que resulta muy importante para la protección de los menores en estas familias en riesgo.

Ahora bien, tanto en madres como en padres, el factor que más destaca es el del área de desarrollo personal y resiliencia. Como se ha señalado en reiteradas ocasiones la tarea vital de ser padres se inscribe en las coordenadas del desarrollo personal adulto y se nutre de éste ( $v g$. Rodrigo, Máiquez, Martín y Byrne, 2008), de modo que una grave carencia en dicho desarrollo tiene graves repercusiones en el ejercicio de las responsabilidades parentales. Así pues, el tener buen autoconcepto y autocontrol, capacidad para resolver problemas y hacerse un proyecto de vida es parte de la tarea de hacerse adulto. Por la misma razón, las capacidades resilientes, como mostrarse asertivo, capaz de autocorrección, perseverante y flexible ante las dificultades cobran una importancia singular en estos contextos de riesgo (Luthar, 2003; Rodrigo et al., 2010b; Walsh, 2004).

No obstante, también es el factor de desarrollo personal y resiliencia el que parece mostrar mayores diferencias entre padres y madres. Los padres de las familias en riesgo muestran algunas competencias asociadas a una visión positiva de la vida (sentido del humor, visión positiva del niño y la familia y tener expectativas positivas hacia el futuro de los niños) y otras relacionadas con la capacidad para el cambio (Capacidad para promover y llegar a acuerdos con la pareja, la motivación para el cambio). En las madres, aparece un grupo de competencias que podrían ser beneficiosas en contextos complejos de riesgo como son el tener un sentido de control sobre los acontecimientos de la vida, la capacidad para responder a múltiples tareas y retos, entrenar la demora de la gratificación en su propia vida, hacer un uso adecuado de la medicación y las estrategias de afrontamiento ante situaciones de estrés. Aquí se dibuja el papel decisivo de la madre evitando perder el norte ante las dificultades que puedan aparecer para la familia, mientras que en el padre se dibuja la necesidad de una visión positiva de la vida que no resulte obstaculizador para el progreso de la misma y la capacidad de llegar a acuerdos con su pareja.

Por último, los factores de integración comunitaria y los de búsqueda de apoyo son muy similares entre ambos progenitores con la particularidad de que en este último factor los padres incorporan la capacidad para colaborar con los profesionales e instituciones que les quieren ofrecer apoyo y ayuda. Es significativo que los servicios sociales no suelan confiar en la colaboración del padre (Dubowitz, 2009; 2006; Rosenberg y Wilcox, 2006), por ello aparece en esta escala como una competencia clave junto con la posibilidad de cambio durante la intervención familiar.

A pesar de lo prometedor de los resultados, hemos de advertir que aún queda mucho trabajo por realizar. Faltaría por determinar la validez externa de este instrumento, analizando la relación que las competencias parentales guardan con otras variables que, tanto en la literatura empírica como en el trabajo cotidiano con familias en riesgo, aparecen como posibles correlatos, tales como el nivel de riesgo de la familia, el nivel de impacto de la situación en el desarrollo del menor, 
o el pronóstico del caso, entre otras. Tampoco disponemos de pruebas para saber si lo que evalúa el educador de familia está en sintonía con lo que el propio padre o madre considera como nivel competencial en las distintas dimensiones evaluadas en el cuestionario. Por último, está por comprobarse la utilidad de la escala en otros contextos de protección del menor como puede ser en los procesos de acogimiento de familia extensa o ajena o en los de adopción.

En conclusión, a la luz de los resultados obtenidos puede afirmarse que esta escala es un instrumento de medida fiable que permite explorar, a partir de un único instrumento, un conjunto amplio de competencias parentales en contextos de riesgo psicosocial. En consonancia con el enfoque de la parentalidad positiva, esta escala resulta un instrumento diagnóstico de valoración de las fortalezas de la familia de cara a planificar el plan de caso y orientar la intervención. Asimis-

\section{Referencias}

Abidin, R. R. (1983). Parenting Stress Index Manual. Charlotesville, VA: Pediatric Psychology Press.

Asparouhov T. y Muthén B. (2009). Exploratory Structural Equation Modeling. Structural Equation Modeling, 16, 397-438.

Asparouhov T. y Muthén B. (2010). Multiple Imputation with Mplus. Recuperado de http:// www.statmodel.com/download/Imputations7.pdf

Azar, S. T. (1998). Parenting and child maltreatment. En I. E. Sigel and K. A. Renninger (Volume 4. Eds.), Child Psychology in practice (pp. 361-388). Handbook of Child Psychology. (5 $5^{\text {th }}$ edition) (W. Damon, Ed.). New York: John Wiley y Sons.

Azar, S. T. y Cote, L. R. (2002). Sociocultural issues in the evaluation of the needs of children in custody decision making: What do our current frameworks for evaluating parenting practices have to offer? International Journal of Law and Psychiatry, 25, 193-217.

Barber, J. G., y Delfabbro, P. H. (2000). The assessment of parenting in child protection cases. Research in Social Work Practice, 10, 243-256.

Barudy J. y Dantagnan M. (2010a). Los desafíos invisibles de ser madre o padre. Manual de evaluación de las competencias y la resiliencia parental. Barcelona: Gedisa editorial.

Barudy J. y Dantagnan M. (2010b). Guia de evaluación de competencias parentales. Recuperado de http://j.mp/wCvsKn

Bayot, A. y Hernández J. V. (2008). Evaluación de la competencia parental. Madrid. CEPE. S. L.

Brown, B. M. (2006). Confirmatory factor analysis for applied research. New York: Guildford.

Byrne B. M. (2010). Structural Equation Modeling with AMOS. Basic concepts, applications and programming. Second Edition. New York: Routledge Taylor and Francis Group..

Comité de Ministros a los Estados Miembros: Recomendación Rec 19 (2006). Politicas de apoyo al ejercicio positivo de la parentalidad. Recuperado de http://www.msps.es/politicaSocial/familiasInfancia/docs/recomendac ion.pdfhttp://www.coe.int/t/dg3/youthfamily

Daly, M. (2007). Parenting in contemporary Europe: a positive approach. Strasbourg: Council of Europe Publications.

De Paúl, J., y Arruabarrena, M. I. (1999). Escalas de Binestar Infantil de Magura y Moses. Un primer estudio para la validación de la versión española. Intervención Psicosocial. Revista sobre Igualdad y Calidad de Vida, 8, 89-107.

Department of Health, Department for Education and Employment and Home Office (2000). Framework for the Assessment of Children in Need and their Families. London: The Stationery Office.

Díaz-Herrero A., Brito de la Nuez A., López P. J. A., Pérez-López J. Y Martínez-Fuentes, M. T. (2010). Estructura factorial y consistencia interna de la versión española del Parenting Stress Index-Short Form. Psicothema, 22, 1033-1038. mo, puede resultar especialmente útil en contextos de educación de padres y madres para el fomento de sus competencias parentales así como en aquellos programas educativos basados en visitas domiciliarias para determinar una línea base a partir de la cual analizar la eficacia de la intervención.

Agradecimientos.- Expresamos nuestro agradecimiento a la Dirección General de Protección del Menor y la Familia de la Comunidad Autónoma de Canarias, a la Consejería de Política Social y Sociosanitaria del Cabildo de Gran Canaria por su apoyo en esta investigación. Asimismo a los técnicos de los equipos de riesgo de la isla de Gran Canaria por participar y colaborar con entusiasmo en la recogida de datos. Por último, agradecemos el apoyo del Ministerio de Ciencia e Innovación mediante el proyecto (TRA2009_0145) concedido al cuarto autor.

Dubowitz H. (2006). Where's dad? A need to understand father's role in child maltreatment. Child Abuse and Neglect, 30, 461-465.

Dubowitz H. (2009). Commentary on fathers and children and maltreatment. Child Maltreatment, 14, 291-293.

Elousa, P. (2003). Sobre la validez de los tests. Psicothema, 15, 315-321.

Elousa P. O. y Zumbo, B. D. (2008). Coeficientes de fiabilidad para escalas de respuesta categórica ordenada. Psicothema, 20, 896-901.

Flora D. B. y Curran P. J. (2004). An empirical evaluation of alternative methods of estimation for confirmatory factor analysis with ordinal data. Psychological methods, 9, 466-491.

Gómez M. E. (2010). El desafío de evaluar familias desde un enfoque ecosistémico: nuevos aportes a la confiabilidad y validez de las escalas NCFAS. En L. Lira (Ed.), Familia y Diversidad (pp. 95-126). Santiago de Chile: Fundación San José para la Adopción.

Hayashi, K., Bentler, P. M. y Yuan, K. H. (2007). On the likelihood ratio test for the number of factors in exploratory factor analysis. Structural Equation Modeling, 14, 505-526.

Hu, L. y Bentler, P. M. (1999). Cutoff criteria for fit indexes in covariance structure analysis: Convectional criteria versus new alternatives. Structural Equation Modeling, 6, 1-55.

Johnson M. A., Stone S., Lou C., Vu, C., Ling J., Mizrahi P. y Austin M. (2006). Family Assessment in Child Welfare Services: Instrument comparisons. En

http://cssr.berkeley.edu/bassc/public/BASSC_FamilyAssessment_FU LL_REPORT091406.pdf

Johnston, Ch. y Mash, E.J. (1989). A measure of parenting satisfaction and efficacy. Journal of Clinical Child Psychology, 18, 167-175.

Lamb, M. E. (2010). The role of the father in child development. New Jersey: John Wiley and Sons, Inc.

Kline, R. B. (2011). Principles and practice of structural equation modeling (3rd Ed.). New York: Guilford press.

Lerman R. I. (2010). Capabilities and Contributions of Unwed Fathers. The Future of Children, 20, 63-85

Lewis, C. y Lamb, M. E. (2003). Fathers' influences on children's development: the evidence from two-parent families. European Journal of Psychology of Education. 17, 211-228.

Lozano, L. M., García-Cueto, E. y Muñiz, J. (2008). Effect of the number of response categories on the reliability and validity of rating scales. Methodology, 4, 73-79.

Luthar, S. S. (2003). Resilience and vulnerability. Adaptation in the context of childhood adversities. New York: Cambridge University Press.

Máiquez, M. L., Rodrigo, M. J., Capote, C. y Vermaes, I. (2000). Aprender en la vida cotidiana. Un programa experiencial para padres. Madrid: Visor.

Magura, S., y Moses, B. (1986). Outcome measures for child welfare services. Washington, D.C.: Child Welfare League of America. 
Marsh H.W., Muthén B., Asparouhov T., Lüdtke, O. Robitzsch A. Morin A. y Trautwein U. (2009). Exploratory structural equation modeling, integrating CFA and EFA: Application to students' evaluations of University Teaching. Structural Equation Modeling, 16, 439-476.

Masten, A. S., y Curtis, W. J. (2000). Integrating competence and psychopathology: Pathways toward a comprehensive science of adaption in development. Development and Psychopathology, 12, 529-550.

Menéndez, S., Jiménez, L. e Hidalgo, M. V. (en prensa). Estructura factorial de la escala PSOC (Parental Sense of Competence) en una muestra de madres ususarias de servicios de preservación familiar. Revista Iberoamericana de Diagnóstico y Evaluación.

Minuchin, P., Colapinto, J. y Minuchin, S. (2000). Pobreza, institución y familia. Buenos Aires: Ed. Amorrortu.

Muthén, B. O., du Toit, S. H. C., y Spisic, D. (1997). Robust inference using weighted least squares and quadratic estimating equations in latent variable modeling with categorical and continuous outcomes. Recuperado de www.gseis.ucla.edu/faculty/muthen/articles/Article_075.pdf

Muthén, L. K. y Muthén B. O. (1998-2010). Mplus User's Guide. Sixth Edition. Los Angeles, CA: Muthén y Muthén.

Reed-Ashcraft, K., Kirk, R. S. y Fraser, M. W. (2001). The reliability and validity of the North Carolina Family Assessment Scale. Research on Social Work Practice, 11(4), 503-520.

Reder, P., Duncan, S. y Lucey, C. (2003). Studies in the assessment of parenting. New York: Routlegde.

Rodrigo, M. J., Máiquez, M. L., Correa, A. D., Martín, J. C., y Rodríguez, G. (2006). Outcome evaluation of a community centre-based program for mothers at high psychosocial risk. Child Abuse and Neglect, 39, 10491064.

Rodrigo, M. J., Máiquez M. L., Martín J. C. y Byrne S. (2008). Preservación Familiar. Un enfoque positivo para la intervención con familias. Madrid: Pirámide.

Rodrigo, M. J., Martín J. C. Cabrera, E. y Máiquez, M. L. (2009). Las competencias parentales en contextos de riesgo psicosocial. Intervención Psicosocial, 18, 113-120.

Rodrigo, M. J., Máiquez M. L. y Martín J. C. (2010a). Parentalidad positiva ) politicas locales de apoyo a las familias. Madrid: Publicaciones de la Federación Española de Municipios y Provincias (FEMP).

Rodrigo, M. J., Máiquez M. L. y Martín J. C. (2010b). La educación parental como recurso psicoeducativo para promover la parentalidad positiva. Madrid: Publicaciones de la Federación Española de Municipios y Provincias (FEMP).

Rose, W. (2010). The Assessment Framework. En Horwath J. (Ed.), The Child's World. The comprehensive Guide to Assessing Children in Need (pp. 34 55). Second Edition. London: Jessica Kingsley Publishers.
Rosenberg, J. y Bradford, W. (2006). The importance of fathers in the healthy development of children. U. S. Children's Bureau Office on Child Abuse and Neglect. Recuperado de http://www.childwelfare.gov/pubs/usermanuals/fatherhood/fatherho od.pdf

Rosenberg, J., y Wilcox, W. B. (2006). The Importance of Fathers in the Healthy Development of Children. Child Abuse and Neglect User Manual Series. Washington, DC: U.S. Department of Health and Human Services, Administration on Children, Youth, and Families, Office on Child Abuse and Neglect.

Schene, P. (2005). Comprehensive Family Assessment Guidelines for Child Welfare. National Child Welfare Resource Center for Family-Centered Practice. Recuperado http://www.acf.hhs.gov/programs/cb/pubs/family_assessment/

Schmitt T. A. (2011). Current methodological considerations in exploratory and confirmatory factor analysis. Journal of Psychoeducational Assessment, 29, 304-321.

Stapleton, L. M. (2006). An assessment of practical solutions for structural equation modeling with complex sample data. Structural Equation Modeling, 13, 28-58.

Tabachnick, B. G. y Fidell, L. S. (2007). Using Multivariate Statistics. Fifth Edition. Boston: Pearson Educations, Inc.

Tamis-Lemonda C. y McFadden K. (2010). Fathers from low-income backgrounds: Myths and Evidence. En Michael E. Lamb (Ed.), The Role of the father in child development (pp. 296-318). New Jersey: John Wiley and Sons, Inc.

Walsh, F. (2004). Resiliencia Familiar. Estrategias para su fortalecimiento. Buenos Aires: Amorrortu editores.

Waters, E. y Sroufe, L.A. (1983). Social competence as a developmental construct. Developmental Review, 3, 79-97.

White, A. (2005). Assessment of parenting capacity. Literature review. Centre for Parenting and Research. Ashfield, Australia: Department of Community Services.

Worthington, R. L. y Whittaker, T. A. (2006). Scale development research: A content analysis and recommendations for best practices. The Counseling Psychologist, 34, 806-838

Yang, Y. y Green, S. B. (2010). A note on structural equation modelling estimates of reliability. Structural Equation Modeling, 17, 66-81.

Yárnoz, S. (2006). ¿Seguimos descuidando a los padres? El papel del padre en la dinámica familiar y su influencia en el bienestar psíquico de sus componentes. Anales de Psicología, 22, 175-185.

(Artículo recibido: 15-4-2012; revisado: 15-9-2012; aceptado: 4-9-2012 